\title{
Nonlinear exciton drift in piezoelectric two-dimensional materials
}

\author{
Vanik Shahnazaryan* \\ ITMO University, St. Petersburg 197101, Russia \\ Habib Rostami ${ }^{\dagger}$ \\ Nordita, KTH Royal Institute of Technology and Stockholm University, Hannes Alfvéns väg 12, 10691 Stockholm, Sweden
}

(Received 13 January 2021; accepted 29 June 2021; published 4 August 2021)

\begin{abstract}
The noncentrosymmetric nature of single-layer (SL) transition-metal dichalcogenides (TMD) manifests itself in the finite piezoelectricity and valley-Zeeman coupling. We microscopically model nonlinear exciton transport in nanobubbles of SL TMDs. Thanks to the giant piezoelectric effect, we obtain an enormous internal electric field, $E_{\text {piezo }} \sim 10^{7} \mathrm{~V} / \mathrm{m}$, resulting in a built-in dipole moment of excitons. We demonstrate that the piezo-induced dipole-dipole interaction provides a novel channel for the nonlinear exciton transport distinct from the conventional isotropic funneling of excitons and leads to the formation of a hexagon-shaped exciton droplet on the top of circularly symmetric nanobubbles. Moreover, we found that the hexagonal distribution of exciton density is preserved even for strongly elliptic nanobubbles. The effect is tunable via the bubble-size dependence of the piezoelectric field $E_{\text {piezo }} \sim h_{\max }^{2} / R^{3}$ with $h_{\max }$ and $R$ being the bubble height and radius, respectively.
\end{abstract}

DOI: 10.1103/PhysRevB.104.085405

\section{INTRODUCTION}

The single-layer (SL) of transition-metal dichalcogenides (TMDs) represents a flatland for probing exciton-related phenomena [1] owing to the visible frequency range direct band gap. There is growing interest toward exciton transport in SL-TMDs [2-5]. Mechanisms governing the exciton transport include the Seebeck effect [3], phonon drag [4], and spatially resolved Coulomb modulation of exciton energy [5]. Exciton-exciton (XX) interaction (exciton nonlinearity) has a strong impact on the exciton transport in quantum wells [6-10]. However, the many-body renormalization of exciton transport in SL-TMD remains unexplored. Exciton nonlinearity primarily emerges in the optical response through a blue shift in the exciton resonances [11]. In unpolarized exciton gas the dominant interaction channel is the short-range exchange [12] which is also the case for pristine SL-TMDs [13-17]. However, in case the excitons possess a permanent dipole moment, the leading scattering channel is the longrange dipole-dipole interaction [18-20]. The dipole moment can be induced in TMD excitons via an external electric field [21-23] and in spatially indirect excitons in bilayers [24,25]. Here we discuss a novel mechanism for inducing an exciton dipole moment in noncentrosymmetric SL-TMD due to strain. Two-dimensional (2D) materials are very flexible to out-ofplane deformation and strong to in-plane stretch [26,27].

\footnotetext{
*vanikshahnazaryan@gmail.com

†habib.rostami@su.se
}

Published by the American Physical Society under the terms of the Creative Commons Attribution 4.0 International license. Further distribution of this work must maintain attribution to the author(s) and the published article's title, journal citation, and DOI. Funded by Bibsam.
This unique property leads to nanobubble formation in graphene and TMDs with a wide radius range $10-500 \mathrm{~nm}$. The elastic stability of nanobubbles enforces a universal aspect ratio of $h_{\max } / R \sim 0.1-0.2$ [28,29], where $h_{\max }$ and $R$ stand for the height and radius of the bubble, respectively. The nonuniform strain in nanobubbles leads to spatial band gap modulation [30], serving as an effective driving potential resulting in the exciton funneling effect $[31,32]$. The funneling manifests itself in a set of phenomena, such as the exciton nanoscale localization [33], strongly enhanced and localized photoluminescence [34], and simultaneous direct and indirect band gap photoluminescence $[29,35]$.

A manifestation of broken inversion symmetry in SLTMDs is the giant piezoelectric constant [36,37]. Lattice deformation can displace the electronic Wannier centers relative to background positive charge resulting in an induced polarization in electrically insulating systems. In SL-TMDs with hexagonal symmetry in the $x y$ plane, the piezoelectric polarization is given by $[38,39]$

$$
\mathbf{P}(\mathbf{r})=\gamma_{\text {piezo }} \mathcal{A}(\mathbf{r}) \times \hat{\mathbf{z}},
$$

where $\mathbf{r}$ is the in-plane position coordinate, $\gamma_{\text {piezo }}$ is the piezoeletric constant, and $\mathcal{A}$ is a fictitious gauge field given in terms of strain tensor component $\left(\mathcal{A}_{x}, \mathcal{A}_{y}\right)=\left(u_{x x}-\right.$ $\left.u_{y y},-2 u_{x y}\right)[39,40]$ similar to the case of graphene [41]. The piezoelectric constant conveys topological information about the valley-Chern number [39,42]. Another consequence of broken inversion symmetry in SL-TMDs is the valleyZeeman effect in the presence of an external magnetic field [43-48]. Interestingly, an inhomogeneous strain profile results in a pseudomagnetic field $\mathbf{B}^{(\tau)}=\partial_{\mathbf{r}} \times \mathbf{A}^{(\tau)}$ changing sign in two valleys $(\tau= \pm)$ at hexagonal Brillouin zone corner owing to the time-reversal symmetry, $\mathbf{A}^{(\tau)}=\tau\left(\beta \hbar / 2 a_{0} e\right) \mathcal{A}$, where $\beta \sim 3$ is the Gruneisen parameter representing 

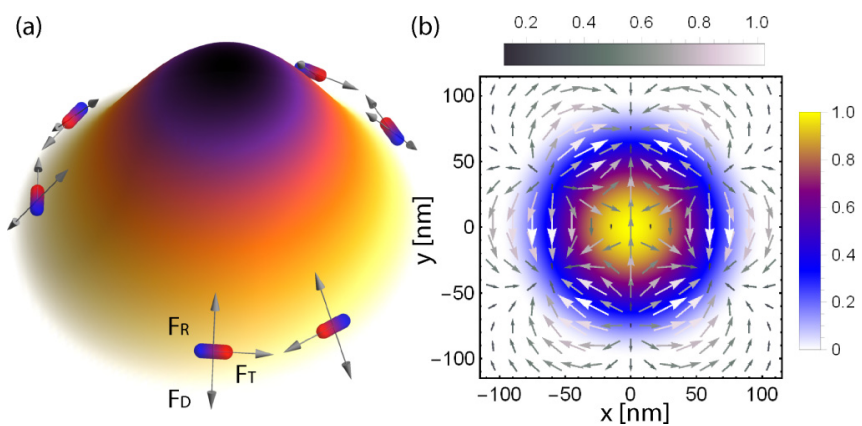

FIG. 1. (a) The sketch of the SL-TMD nanobubble. The straininduced band gap modulation gives rise to radial funneling force, which is partially compensated by the counteracting diffusive propagation. The piezoelectricity-induced dipolar interaction and the emergent pseudomagnetic field generate highly anisotropic forces, leading to spatially inhomogenous drift of excitons. (b) The electric field streamlines on top of bubble profile colormap. The horizontal (vertical) colorbar corresponds to piezo-induced electric field, $E_{\text {piezo }} / E_{0}$ with $E_{0}=10^{7} \mathrm{~V} / \mathrm{m}$ (height $h / h_{\max }$ ).

electron-lattice coupling and $a=\sqrt{3} a_{0}$ is the lattice constant, with $a=3.18 \AA$.

In this article, we utilize the interplay of $\mathrm{XX}$ interaction, strong piezoelectricity, and nanobubble formation in TMD materials to study the piezoelectricity-driven nonlinear exciton transport in SL-TMD. The considered system is schematically depicted in Fig. 1(a). Nonuniform strain induces a bound charge density $\rho_{\text {piezo }}(\mathbf{r})=-\partial_{\mathbf{r}} \cdot \mathbf{P}(\mathbf{r})$ that can generate a piezoeletric field based on the Poisson's equation:

$$
\partial_{\mathbf{r}} \cdot \epsilon_{0} \mathbf{E}_{\text {piezo }}(\mathbf{r})=\rho_{\text {piezo }}(\mathbf{r}) \delta(z),
$$

where $\epsilon_{0}$ is the vacuum permittivity. Equation (2) should be supplemented by Faraday's law $\partial_{\mathbf{r}} \times \mathbf{E}_{\text {piezo }}=0$ enforcing the static character of the emergent electric field. A giant piezoelectric effect yields in an enormous internal electric field of the order of $\sim 10^{7} \mathrm{~V} / \mathrm{m}$, depicted in Fig. 1(b), which induces an exciton dipole moment $\mathbf{d}(\mathbf{r})=\alpha_{E} \mathbf{E}_{\text {piezo }}(\mathbf{r})$, where $\alpha_{E}$ stands for the exciton polarizability. Utilizing the highly tunable piezo-induced dipole moment can be a novel framework for realizing many-body-driven exciton physics. Here, we microscopically develop a theory of exciton transport in strained SL-TMDs accounting for the long-range dipolar XX interaction. Owing to the exciton nonlinearity, we predict a long-standing spatially threefold symmetric exciton density which can be directly accessed via photoluminescence measurements.

\section{METHOD}

\section{A. Microscopic theory of exciton transport}

The 2D exciton dynamics is characterized by the Boltzmann distribution function $f_{\mathbf{p}}(\mathbf{r}, t)$, where $\mathbf{p}$ is the exciton momentum. Accordingly, the exciton transport is modeled based on the Vlasov-Boltzmann kinetic equation:

$$
\begin{aligned}
& \partial_{t} f_{\mathbf{p}}(\mathbf{r}, t)+\mathbf{v}_{\mathbf{p}} \cdot \partial_{\mathbf{r}} f_{\mathbf{p}}(\mathbf{r}, t)+\mathbf{F}(\mathbf{r}, t) \cdot \partial_{\mathbf{p}} f_{\mathbf{p}}(\mathbf{r}, t) \\
& \quad=-f_{\mathbf{p}}(\mathbf{r}, t) / \tau_{d}+Q\left(f_{\mathbf{p}}\right),
\end{aligned}
$$

where $\tau_{d}, \mathbf{v}_{\mathbf{p}}=\mathbf{p} / M$ are the exciton lifetime and velocity, respectively, with $M$ being the exciton mass. The effective force

$$
\mathbf{F}(\mathbf{r}, t)=-\partial_{\mathbf{r}} V(\mathbf{r}, t)
$$

in which

$$
V(\mathbf{r}, t)=V_{\mathrm{X}}^{\mathrm{R}}(\mathbf{r})+V_{\mathrm{X}}^{\mathrm{B}}(\mathbf{r})+V_{\mathrm{XX}}(\mathbf{r}, t)
$$

denotes the instantaneous potential energy acting on excitons at position $\mathbf{r}$. The effective potential includes the exciton energy spatial modulation due to the strain-induced band gap renormalization, $V_{\mathrm{X}}^{\mathrm{R}}(\mathbf{r})$; the valley-Zeeman shift due to the pseudomagnetic field, $V_{\mathrm{X}}^{\mathrm{B}}(\mathbf{r})$; and the long-range dipoledipole XX interaction,

$$
V_{\mathrm{XX}}(\mathbf{r}, t)=\mathbf{E}_{d}(\mathbf{r}, t) \cdot \mathbf{d}(\mathbf{r}) .
$$

The effective electric field $\mathbf{E}_{d}(\mathbf{r}, t)$ acting on excitons at position $\mathbf{r}$ due to the interaction with other excitons follows (see the Appendix A for the details)

$$
\partial_{\mathbf{r}} \cdot \epsilon_{0} \mathbf{E}_{d}(\mathbf{r}, t)=-\partial_{\mathbf{r}} \cdot[\mathbf{d}(\mathbf{r}) n(\mathbf{r}, t) \delta(z)],
$$

where $n(\mathbf{r}, t)=\sum_{\mathbf{p}} f_{\mathbf{p}}(\mathbf{r}, t)$ stands for the exciton density. For a temporally slow varying electric field $\partial_{\mathbf{r}} \times \mathbf{E}_{d}(\mathbf{r}, t) \approx 0$, while for the exciton polarization density $\partial_{\mathbf{r}} \times[\mathbf{d}(\mathbf{r}) n(\mathbf{r}, t) \delta(z)]$ does not necessarily vanish.

Contrary to the real magnetic field, the pseudomagnetic field results in a normal Zeeman correction to the exciton energy owing to the absence of inversion and the presence of time-reversal symmetry. Considering the conventional radial funneling potential owing to the band gap renormalization that is proportional to the trace of strain tensor, we write the corresponding energy modulation as follows:

$$
\begin{gathered}
V_{\mathrm{X}}^{\mathrm{R}}(\mathbf{r})=g_{0} \sum_{\ell} u_{\ell \ell}(\mathbf{r}), \\
V_{\mathrm{X}}^{\mathrm{B}}(\mathbf{r})=g_{\mathrm{VZ}} \mu_{B} B(\mathbf{r}) .
\end{gathered}
$$

Here $\mu_{B}$ is the Bohr magneton, and we set the valleyZeeman coupling $g_{\mathrm{VZ}} \approx-0.81$ [48] and the radial funneling strength $g_{0} \approx 300 \mathrm{meV}$ [49]. We neglect the diamagnetic shift in Eq. (8) as for the ground state exciton it is negligibly small compared with valley Zeeman effect [50]. The collision term $Q\left(f_{\mathbf{p}}\right)$, which models the dephasing processes, is treated within a phenomenological relaxation time approximation $Q\left(f_{\mathbf{p}}\right)=\left[\bar{f}_{\mathbf{p}}(\mathbf{r}, t)-f_{\mathbf{p}}(\mathbf{r}, t)\right] / \tau_{C}$, with $\tau_{C}$ denoting the collision time and $\bar{f}_{\mathbf{p}}(\mathbf{r}, t)$ following the quasiequilibrium Maxwell-Boltzmann distribution. Utilizing a ChapmanEnskog type ansatz [51] and performing the momentum integration, we obtain (see the Appendix B for the details of derivation)

$$
\partial_{t} n(\mathbf{r}, t)=\partial_{\mathbf{r}} \cdot\left[D \partial_{\mathbf{r}} n(\mathbf{r}, t)-\mu n(\mathbf{r}, t) \mathbf{F}(\mathbf{r}, t)\right]-\frac{n(\mathbf{r}, t)}{\tau_{d}} .
$$

Here $D=\mu k_{\mathrm{B}} T$ stands for the diffusion coefficient with $T$ being the lattice temperature, $\mu=\tau_{\text {eff }} / M$ is the mobility in which

$$
\frac{1}{\tau_{\text {eff }}}=\frac{1}{\tau_{C}}+\frac{1}{\tau_{d}}
$$


is an effective relaxation rate. Accordingly, the exciton mobility depends on collision time as well as the finite lifetime, while previously its dependence on the lifetime was underestimated.

\section{B. Characteristics of circular nanobubble in TMDs}

Exact solution of displacement profile for SL-TMD circular nanobubble strongly depends on the elastic boundary conditions and external mechanical force distribution [39,52-55]. However, using an intuitive perspective, we can estimate a basic analytic solution for the pure bending approximation, where the bending (curvature) energy dominates the elasticstretching energy [39]. The out-of-plane displacement is thus given as

$$
h(r)=h_{\max }\left(1-\frac{r^{2}}{R^{2}}\right)^{2} \Theta(R-r),
$$

where $R\left(h_{\max }\right)$ is the bubble radius (height). We set the aspect ratio factor $\xi=h_{\max } / R=0.2$. To ensure the elastic stability there is also a radial displacement $u(r)$ to be determined. Considering circular symmetry of the bubble one can evaluate strain tensor elements in the polar coordinate as

$$
\begin{aligned}
& u_{r r}=\partial_{r} u(r)+\left[\partial_{r} h(r)\right]^{2} / 2, \\
& u_{\theta \theta}=u(r) / r,
\end{aligned}
$$

and $u_{r \theta}=u_{\theta r}=0$. Utilizing linear elasticity formalism [39], the radial displacement reads $u(r)=u_{0}\left(4 \alpha-7 \alpha^{7}-20 \alpha^{5}-\right.$ $\left.18 \alpha^{3}\right)$ for $\alpha \leqslant 1$ and $u(r)=-u_{0} / \alpha^{2}$ for $\alpha>1$ where $\alpha=$ $r / R$ and $u_{0}=\xi h_{\max } / 6$. The corresponding bubble profile is shown in Fig. 1(b). Eventually, the trace of strain tensor reads $\sum_{\ell} u_{\ell \ell}=u_{r r}+u_{\theta \theta}$. Having strain tensor components, we obtain pseudo-gauge vector $\mathcal{A}(\mathbf{r})$ and thus the piezoelectric charge density follows

$$
\rho_{\text {piezo }}(\mathbf{r})=-\partial_{\mathbf{r}} \cdot \mathbf{P}(\mathbf{r})=C \frac{\gamma_{\text {piezo }}}{R} \rho\left(\frac{r}{R}\right) \sin (3 \theta),
$$

where $C=-4 \xi^{2} / 3$ and $\rho(\alpha)$ is a dimensionless function:

$$
\rho(\alpha)=\left\{\begin{array}{ll}
\alpha^{3}\left(4-3 \alpha^{2}\right) & \text { if } \alpha \leqslant 1 \\
1 / \alpha^{3} & \text { if } \alpha>1
\end{array} .\right.
$$

Plugging piezo-induced charge density into the Poisson equation given in Eq. (2), we evaluate the built-in electric field due to piezoelectricity. The resulting electric field is depicted in Fig. 1(b). Remarkably, it has a threefold symmetry and is of the order of $E_{\text {piezo }} \sim 10^{7} \mathrm{~V} / \mathrm{m}$, in line with with a recent experimental report of piezoelectricity in TMD nanobubbles [56]. Finally, it is easy to show that $\partial_{\mathbf{r}} \cdot[\mathcal{A}(\mathbf{r}) \times \hat{\mathbf{z}}]=$ $\hat{\mathbf{z}} \cdot\left[\partial_{\mathbf{r}} \times \mathcal{A}(\mathbf{r})\right]$, which implies

$$
B(\mathbf{r})=\frac{\beta \hbar}{2 a_{0} e} \frac{\rho_{\text {piezo }}(\mathbf{r})}{\gamma_{\text {piezo }}} .
$$

\section{RESULTS AND DISCUSSION}

We numerically simulate Eq. (10) to study nonlinear exciton transport in SL-TMDs. The parameters are chosen as collision time $\tau_{C}=0.26$ ps [57], piezelectric constant $\gamma_{\text {piezo }}=2.9 \times 10^{-10} \mathrm{C} / \mathrm{m}[36,37]$, and exciton polarizability $\alpha_{E}=5 \times 10^{-18} \mathrm{eV}(\mathrm{m} / \mathrm{V})^{2}$ [21]. The exciton lifetime scales linearly with temperature, i.e., $\tau_{d} \propto T$ [58], and for SL $\mathrm{MoS}_{2}$ it can be approximated as $\tau_{d} \approx(0.1+0.9 T[\mathrm{~K}])$ ps [59]. We set the radius of the bubble as $R=100 \mathrm{~nm}$ and assume an initial exciton population density as $n(\mathbf{r}, 0)=$ $n_{\max } e^{-\left|\mathbf{r}-\mathbf{r}_{0}\right|^{2} / \Delta^{2}}$, where we set $\Delta=R, n_{\max }=10^{13} \mathrm{~cm}^{-2}[60]$, with the center of pump spot coinciding with the bubble center, i.e., $\mathbf{r}_{0}=0$.

Figure 2 demonstrates the drifting potentials illustrating the conventional radial funneling, the trigonal symmetric Zeeman coupling and XX interaction, respectively, in panels (a), (b), and (c). The XX-interaction potential originates from the asymmetric profile of piezoelectric induced electric field depicted in Fig. 1(b). The corresponding force vector lines are depicted on each colormap plot. Remarkably, the XXinteraction force is significant close to the center of the bubble, unlike the negligibly small magnetic-fieldinduced force. Considering the universal aspect ratio factor $\xi$, the only control parameter is the bubble radius $R$. Accordingly, we plot the radius dependence of each drifting potential in Fig. 2(d) where it depicts the maximum value of each term. As seen, the drifting potentials scale with the bubble radius as $1 / R^{\eta}$ with $\eta=0,1$ and $\sim 2$ for $V_{\mathrm{X}}^{\mathrm{R}}, V_{\mathrm{X}}^{\mathrm{B}}$, and $V_{\mathrm{XX}}$, respectively. For largesize bubbles, e.g., $R>100 \mathrm{~nm}$, the radial funneling term is the dominant driving force compared to the magnetic and XX-interaction terms. Based on the different scaling of potentials with $R$, in nanobubbles, e.g., $R<100 \mathrm{~nm}$, the magnetic and $\mathrm{XX}$-interaction-induced forces are enhanced to compete with the radial funnel term, leading to an anisotropic exciton density.

The dynamics of exciton transport is presented in Fig. 3, where panels (a), (b), and (c) indicate snapshots of exciton distribution at $T=10 \mathrm{~K}$ corresponding to $t=0,2$ and $10 \mathrm{ps,}$ respectively. As seen, at the intermediate stage, the particle distribution is strongly asymmetric showing a hexagonal profile [see Fig. 3(b)]. At the later time, panel (c), this asymmetry becomes less pronounced due to the finite exciton lifetime. Cross-section plots along $x$ and $y$ directions are depicted in panel (d), which illustrate the asymmetry of exciton density on a circularly symmetric bubble more quantitatively. We adapt notation

$$
n_{ \pm}(\delta, t)=[n(x=\delta, y=0, t) \pm n(x=0, y=\delta, t)] / 2
$$

for the population average $(+)$ and difference $(-)$, respectively. The anisotropic density profile is revealed by a vanishing $n_{-}$at the bubble center and two sharp peaks at the edges of the central hexagon. The distance between two peaks $2 \delta_{0}$ can be a good experimental probe to estimate the size of the hexagon side $\approx \delta_{0}$. Due to a fast initial radial drift of particles to the bubble center, the pseudomagnetic field has a vanishing impact on the particle distribution. Hence, the anisotropic exciton density distribution at the bubble central region is only associated with the $\mathrm{XX}$-interaction effects.

To preserve the asymmetric density distribution for a longer time, we decrease bubble radius to $R=75 \mathrm{~nm}$. The snapshots of evolution are shown in Figs. 3(e)-3(g). One can observe that the hexagonlike distribution lasts for the longer period by tracing the similarity of density profile at $t=2$ ps and $t=10 \mathrm{ps}$ shown in panels (f) and (g), respectively. The long-living asymmetry is clearly visible in looking at 

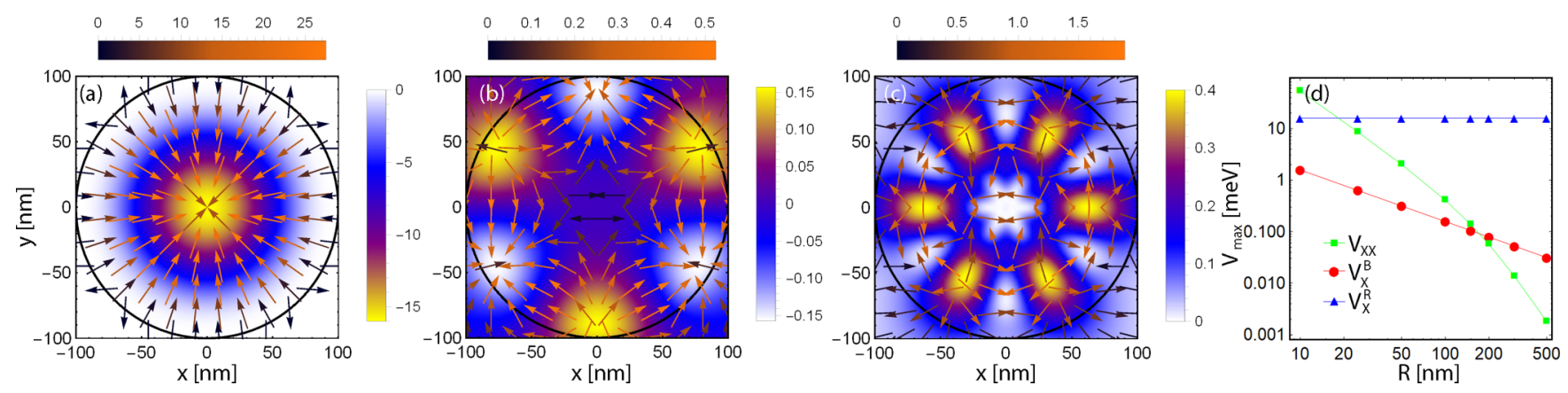

FIG. 2. (a)-(c) The spatial colormap of strain-induced potential energies and the corresponding streamlines of drifting forces: (a) scalar funneling potential; (b) pseudomagnetic field; (c) dipole-dipole interactions. The vertical (horizontal) colorbar corresponds to potential energy in meV (drifting force in $F_{0}=100 \mathrm{meV} / \mathrm{nm}$ ). The black circle denotes the bubble boundary with radius $R=100 \mathrm{~nm}$. (d) Potential energy peak value plotted versus the bubble radius. For macroscopic bubbles $(R>100 \mathrm{~nm})$, the exciton dynamics are solely governed by the radial funneling term while for nanobubbles $(R<100 \mathrm{~nm})$ the dipolar and magnetic sources rapidly increase by reducing the bubble radius.

the time evolution of the population difference along crosssections, cf. panels (h) and (d). The pronounced asymmetry of density distribution is due to the enhancement of $\mathrm{XX}$ interaction for the smaller bubble size. The inhomogeneous distribution of excitons can be retained even longer in time by increasing the temperature $T$, which will result in an increased
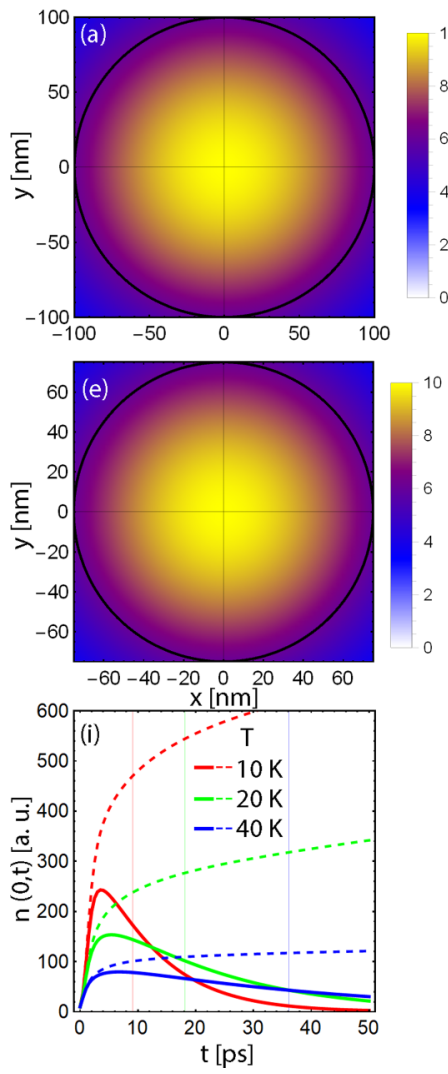
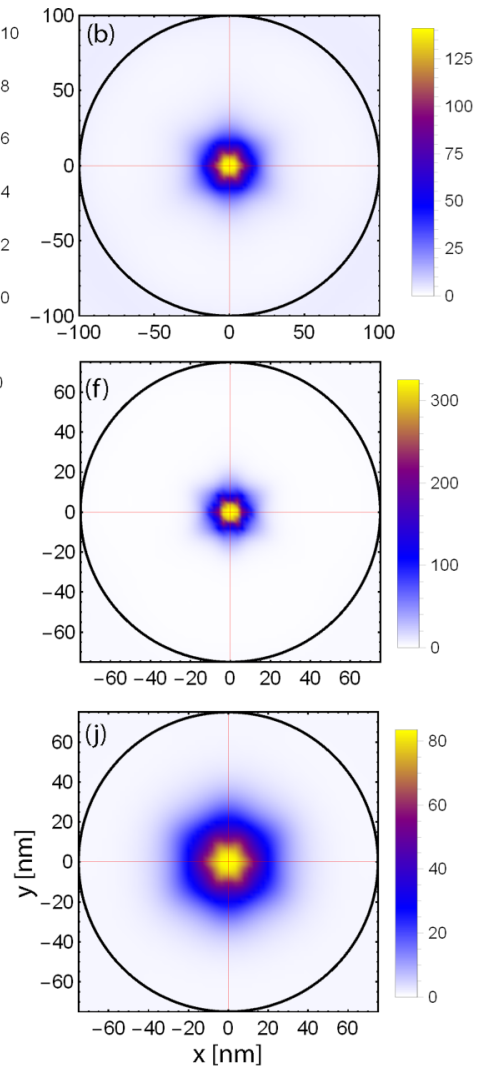
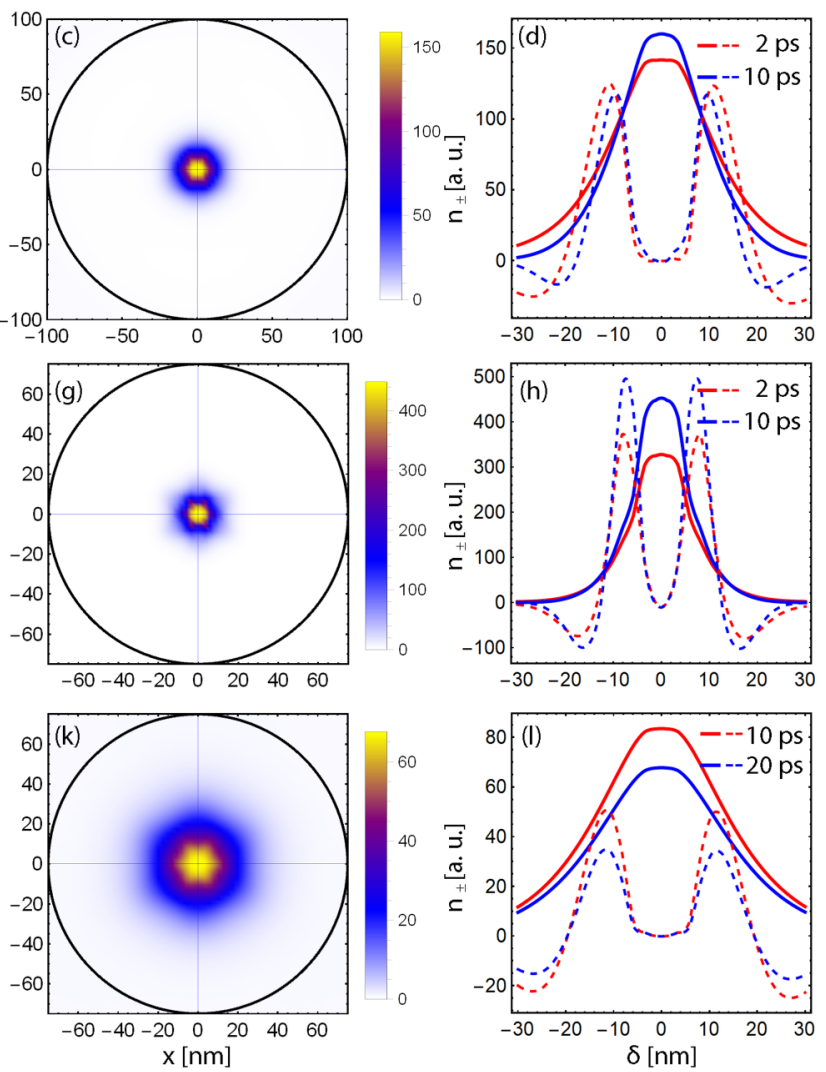

FIG. 3. (a), (b), and (c) Snapshots of exciton density (in a.u.) for $T=10 \mathrm{~K}$ and $R=100 \mathrm{~nm}$ bubble radius at $t=0,2$, and 10 ps, respectively. The thin lines indicate cross-sections along $x$ and $y$ directions. (d) The difference (dashed curves amplified by a factor of 5) and the average (solid curve) of exciton density along $x$ and $y$ cross-sections at different time steps, corresponding to panels (b) and (c). (e), (f) and (g) Snapshots of exciton density (in a.u.) for $T=10 \mathrm{~K}$ and $R=75 \mathrm{~nm}$ bubble radius at $t=0,2$, and $10 \mathrm{ps,} \mathrm{respectively.} \mathrm{In} \mathrm{the} \mathrm{smaller}$ bubble the asymmetric hexagon -shape is pronounced and persists for longer time. (h) The difference (dashed curves amplified by a factor of 5) and the average (solid curve) of exciton density in $x$ and $y$ cross-sections at different time steps, corresponding to panels (f) and (g). (i) Exciton density at the bubble center in the absence of XX-interactions at several temperatures. The solid lines correspond to dissipative dynamics, and the dashed lines correspond to the absence of dissipation, i.e., $\tau_{d} \rightarrow \infty$. Vertical lines indicate the respective lifetime value $\tau_{d}=(0.1+0.9 T[\mathrm{~K}])$ ps. (j), (k) The snapshots of exciton density at $t=10,20 \mathrm{ps}$ with elevated temperature $T=40 \mathrm{~K}$. (l) The difference (dashed curves amplified by a factor of 5) and the average (solid curve) of exciton density in $x$ and $y$ cross-sections at different time steps, corresponding to panels (j) and (k). 

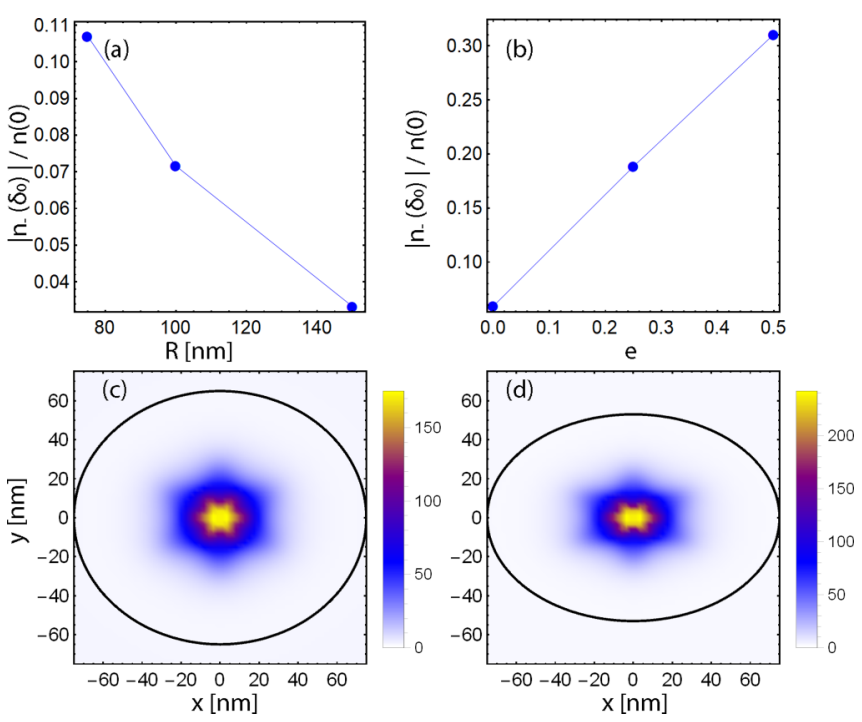

FIG. 4. (a) Hexagon "brightness" versus the nanobubble radius for circular bubble. Here $T=10 \mathrm{~K}$ and $t=10 \mathrm{ps}$. (b) Hexagon "brightness" versus the ellipticity rate. (c), (d) Snapshot of exciton density distribution for ellipticity rate (c) $e=0.25$, (d) $e=0.5$. In panels (b)-(d) $T=40 \mathrm{~K}, t=10 \mathrm{ps}, R=75 \mathrm{~nm}$.

exciton lifetime and enhanced diffusion. The counteroriented diffusion effect (from center to bubble edge) can partially compensate the radial funnel effect (from edge to the bubble center) by altering the temperature. We analyze the dynamics of excitons in the absence of anisotropic forces at different temperatures after ignoring dipole-dipole and magnetic potential energies. The resulting exciton population in the bubble center is shown versus time in Fig. 3(i). Remarkably, in the case when the dissipation is neglected (dashed curves), a temperature-dependent dramatic drop in the exciton density temporal slope is predicted. Moreover, at elevated temperatures such a quasiequilibrium dynamics is reached at timescale far below the exciton lifetime (see blue dashed curve). The corresponding evolution at $T=40 \mathrm{~K}$ is shown in Figs. 3(j)-3(1). Inclined the partial compensation of radial funneling by the diffusion effect, a nearly constant hexagonal shape lasts during the evolution process. The density snapshots at $t=10 \mathrm{ps}$ and $t=20 \mathrm{ps}$ for bubble size $R=75 \mathrm{~nm}$ and temperature $T=40 \mathrm{~K}$ are depicted in panels $(\mathrm{j})$ and $(\mathrm{k})$, respectively. The corresponding cross-section plots are shown in panel (l). As seen, the net effect of raising the temperature is an increase in the central hexagon size with sharper edges which can last for a longer time.

In order to quantitatively unveil the scaling of exciton population anisotropy with bubble radius in Fig. 4(a), we plot the hexagon-normalized population difference $\left|n_{-}\left(\delta_{0}, t\right)\right| / n(0, t)$, referred as hexagon "brightness." Remarkably, the brightness drops by increasing the radius associated with the rapid reduction of the dipole-dipole interactions in large bubbles. Accordingly, it results in a diminished asymmetry of exciton gas distribution.

We further consider the exciton dynamics in an elliptic nanobubble where the circular symmetry is violated. The ellipse equation reads as $x^{2}+y^{2} /(1-e)=R^{2}$, where $e$ is the ellipticity rate. We note that all drifting forces can be rescaled via a transformation to the $X-Y$ coordinate frame as $X=x / R, Y=y /(R \sqrt{1-e})$ and with a subsequent inverse transformation we obtain the expressions in the original $x-y$ frame. The spatial distributions of exciton density for elliptical nanobubbles are shown in Figs. 4(b)-4(d). Evidently, the hexagonal distribution is qualitatively preserved even in a strongly elliptic nanobubble with $e=0.5$. Moreover, as it follows from the Fig. 4(b), the hexagon brightness is strongly enhanced when the ellipticity rate is increased.

\section{SUMMARY AND OUTLOOK}

Piezoelectricity impact on the nonlinear exciton transport in SL-TMD nano-bubble is studied. We develop a nonlinear drift-diffusion theory for excitons starting from the Vlasov-Boltzmann kinetic equation. A strong piezoinduced electric field can polarize excitons resulting in a novel interaction channel being available in the nanobubble systems. Furthermore, we discuss other drifting forces originating from strain-induced band gap renormalization and the valley-Zeeman coupling. In nanobubbles of SL-TMD, the piezo-induced XX interaction is the dominant factor in describing the long-living hexagon-shape of the exciton droplet. The predicted phenomena of hexagon-shaped distribution of exciton density is retained for the nanobubbles with broken circular symmetry as well. The hexagon size and time evolution are controllable via the temperature dependence of diffusion and the bubble radius dependence of the XX interaction. Our technical theory can be generalized in other systems such as heterostructures of 2D materials with different inhomogeneous strain profiles or exhibiting Moiré patterns [61]. Based on the current nanofabrication technology, a lattice of long-range interacting droplets in an array nanobubbles in TMD is designable [62], potentially resulting in a spontaneous long-range coherence.

\section{ACKNOWLEDGMENTS}

H.R. thanks Emmanuele Cappelluti for useful discussions. This work was supported by the Russian Science Foundation (Grant No. 19-72-00171). H.R. acknowledges support from the Swedish Research Council (VR 2018-04252).

\section{APPENDIX A: MEAN-FIELD ELECTRIC FIELD INDUCED BY A DIPOLAR EXCITON GAS}

The charge density of a single dipole reads as

$$
\begin{aligned}
\rho_{j}(\mathbf{r}) & =\lim _{\Delta \mathbf{r}_{j} \rightarrow \mathbf{0}} q\left[\delta\left(\mathbf{r}-\mathbf{r}_{j}-\Delta \mathbf{r}_{j}\right)-\delta\left(\mathbf{r}-\mathbf{r}_{j}\right)\right] \delta(z) \\
& =-\lim _{\Delta \mathbf{r}_{j} \rightarrow \mathbf{0}}\left(q \Delta \mathbf{r}_{j}\right) \cdot \partial_{\mathbf{r}} \delta\left(\mathbf{r}-\mathbf{r}_{j}\right) \delta(z) \\
& \equiv-\mathbf{d}\left(\mathbf{r}_{j}\right) \cdot \partial_{\mathbf{r}} \delta\left(\mathbf{r}-\mathbf{r}_{j}\right) \delta(z) .
\end{aligned}
$$

Notice that $\mathbf{d}\left(\mathbf{r}_{j}\right)=\lim _{\Delta \mathbf{r}_{j} \rightarrow \mathbf{0}}\left(q \Delta \mathbf{r}_{j}\right)$ is the dipole moment located at position $\mathbf{r}_{j}$ and $q$ is a unit of electric charge. Therefore, the electric field created by a single dipole satisfies the following Poisson's equation,

$$
\partial_{\mathbf{r}} \cdot \epsilon_{0} \mathbf{E}\left(\mathbf{r}, \mathbf{r}_{j}\right)=\rho_{j}(\mathbf{r})=-\mathbf{d}\left(\mathbf{r}_{j}\right) \cdot \partial_{\mathbf{r}} \delta\left(\mathbf{r}-\mathbf{r}_{j}\right) \delta(z) .
$$


The total electric field created by an ensemble of dipoles is

$$
\mathbf{E}_{d}(\mathbf{r})=\sum_{j} \mathbf{E}\left(\mathbf{r}, \mathbf{r}_{j}\right)=\int \mathbf{E}\left(\mathbf{r}, \mathbf{r}^{\prime}\right) n\left(\mathbf{r}^{\prime}\right) \mathrm{d} \mathbf{r}^{\prime}
$$

where we used the definition of exciton density $n(\mathbf{r})=$ $\sum_{j} \delta\left(\mathbf{r}-\mathbf{r}_{j}\right)$ with $\sum_{j}$ summing over all excitons. Thus, for the total electric field we reach

$$
\begin{aligned}
\partial_{\mathbf{r}} \cdot \epsilon_{0} \mathbf{E}_{d}(\mathbf{r}) & =\sum_{j} \rho_{j}(\mathbf{r}) \\
& =-\int n\left(\mathbf{r}^{\prime}\right) \mathbf{d}\left(\mathbf{r}^{\prime}\right) \cdot \partial_{\mathbf{r}} \delta\left(\mathbf{r}-\mathbf{r}^{\prime}\right) \delta(z) \mathrm{d} \mathbf{r}^{\prime} \\
& =-\partial_{\mathbf{r}} \cdot \int n\left(\mathbf{r}^{\prime}\right) \mathbf{d}\left(\mathbf{r}^{\prime}\right) \delta\left(\mathbf{r}-\mathbf{r}^{\prime}\right) \delta(z) \mathrm{d} \mathbf{r}^{\prime} \\
& =-\partial_{\mathbf{r}} \cdot[\mathbf{d}(\mathbf{r}) n(\mathbf{r}) \delta(z)] .
\end{aligned}
$$

By replacing the static density $n(\mathbf{r})$ with the instantaneous time-dependent density $n(\mathbf{r}, t)$, we obtain

$$
\partial_{\mathbf{r}} \cdot \epsilon_{0} \mathbf{E}_{d}(\mathbf{r}, t)=-\partial_{\mathbf{r}} \cdot[\mathbf{d}(\mathbf{r}) n(\mathbf{r}, t) \delta(z)],
$$

corresponding to Eq. (7).

\section{APPENDIX B: FROM VLASOV-BOLTZMANN KINETIC THEORY TO NONLINEAR DRIFT-DIFFUSION EQUATION}

In order to derive the drift-diffusion equations, we proceed with dimensionless variables. To do so, we introduce characteristic quantities describing the system. The characteristic velocity is determined by the lattice temperature as $v_{0}=\sqrt{k_{B} T / M}$. Hence, the mean-free path reads as $\lambda=v_{0} \tau_{C}$. The time, which a particle with the typical velocity $v_{0}$ needs to run through the sample, is $\tau_{0}=L / v_{0}$, where $L$ is the length of the sample. One can characterize the system with the reference length $\lambda_{0}=\sqrt{\lambda L}$. Finally, the reference momentum is $p_{0}=M v_{0}=\sqrt{M k_{B} T}$. We introduce dimensionless quantities as $\tilde{\mathbf{r}}=\mathbf{r} / \lambda_{0}, \tilde{V}=V /\left(k_{B} T\right), \tilde{\mathbf{p}}=\mathbf{p} / p_{0}, \tilde{\mathbf{v}}_{\mathbf{p}}=\mathbf{v}_{\mathbf{p}} / v_{0}=\tilde{\mathbf{p}}$, $\sigma=\lambda / \lambda_{0}, \tilde{t}=t / \tau_{0}=t \sigma^{2} / \tau_{C}, \tilde{Q}(f)=\tau_{C} Q(f)$. Then in dimensionless form, the kinetic equation will read as

$$
\begin{aligned}
& \sigma^{2} \partial_{\tilde{t}} f_{\tilde{\mathbf{p}}}(\tilde{\mathbf{r}}, \tilde{t})+\sigma\left[\tilde{\mathbf{p}} \cdot \partial_{\tilde{\mathbf{r}}} f_{\tilde{\mathbf{p}}}-\partial_{\tilde{\mathbf{r}}} \tilde{V}(\tilde{\mathbf{r}}, \tilde{t}) \cdot \partial_{\tilde{\mathbf{p}}} f_{\tilde{\mathbf{p}}}(\tilde{\mathbf{r}}, \tilde{t})\right] \\
& \quad+\frac{\tau_{C}}{\tau_{d}} f_{\tilde{\mathbf{p}}}(\tilde{\mathbf{r}}, \tilde{t})=\tilde{Q}\left[f_{\tilde{\mathbf{p}}}(\tilde{\mathbf{r}}, \tilde{t})\right]
\end{aligned}
$$

We apply a Chapman-Enskog-type ansatz [51] as $f_{\tilde{\mathbf{p}}}(\tilde{\mathbf{r}}, \tilde{t})=\left[n_{0}(\tilde{\mathbf{r}}, \tilde{t}) \bar{f}_{\tilde{\mathbf{p}}}^{0}+\sigma g_{\sigma \tilde{\mathbf{p}}}(\tilde{\mathbf{r}}, \tilde{t})\right] e^{-\frac{\tau_{0}}{\tau_{d}} \tilde{t}}, \quad$ which accounts for the finite lifetime of excitons. Here $\bar{f}_{\tilde{\mathbf{p}}}^{0}=2 \pi e^{-\frac{\left|\tilde{p}^{2}\right|}{2}}$ is the quasiequilibrium Maxwell-Boltzmann distribution, with $\left|\tilde{p}^{2}\right| / 2$ standing for dimensionless kinetic energy, and $g_{\sigma \tilde{\mathbf{p}}}$ is a small correction to homogeneous distribution. Inserting into kinetic equation, we get

$$
\begin{aligned}
& \sigma\left\{\left[\partial_{\tilde{t}} n_{0}(\tilde{\mathbf{r}}, \tilde{t})-\frac{\tau_{0}}{\tau_{d}} n_{0}(\tilde{\mathbf{r}}, \tilde{t})\right] \bar{f}_{\tilde{\mathbf{p}}}^{0}\right. \\
& \left.+\sigma\left[\partial_{\tilde{t}} g_{\sigma \tilde{\mathbf{p}}}(\tilde{\mathbf{r}}, \tilde{t})-\frac{\tau_{0}}{\tau_{d}} g_{\sigma \tilde{\mathbf{p}}}(\tilde{\mathbf{r}}, \tilde{t})\right]\right\} \\
& +\tilde{\mathbf{p}} \cdot \partial_{\tilde{\mathbf{r}}} n_{0}(\tilde{\mathbf{r}}, \tilde{t}) \bar{f}_{\tilde{\mathbf{p}}}^{0}-\partial_{\tilde{\mathbf{r}}} \tilde{V}(\tilde{\mathbf{r}}, \tilde{t}) \cdot \partial_{\tilde{\mathbf{p}}} n_{0}(\tilde{\mathbf{r}}, \tilde{t}) \bar{f}_{\tilde{\mathbf{p}}}^{0} \\
& +\sigma\left[\tilde{\mathbf{p}} \cdot \partial_{\tilde{\mathbf{r}}} g_{\sigma \tilde{\mathbf{p}}}(\tilde{\mathbf{r}}, \tilde{t})-\partial_{\tilde{\mathbf{r}}} \tilde{V}(\tilde{\mathbf{r}}, \tilde{t}) \cdot \partial_{\tilde{\mathbf{p}}} g_{\sigma \tilde{\mathbf{p}}}(\tilde{\mathbf{r}}, \tilde{t})\right] \\
& +\frac{\tau_{C}}{\tau_{d} \sigma} n_{0}(\tilde{\mathbf{r}}, \tilde{t}) \bar{f}_{\tilde{\mathbf{p}}}^{0}+\frac{\tau_{C}}{\tau_{d}} g_{\sigma \tilde{\mathbf{p}}}(\tilde{\mathbf{r}}, \tilde{t})=-g_{\sigma \tilde{\mathbf{p}}}(\tilde{\mathbf{r}}, \tilde{t}),
\end{aligned}
$$

where we use $\tilde{Q}\left(g_{\sigma \tilde{\mathbf{p}}}\right)=-\sigma g_{\sigma \tilde{\mathbf{p}}}(\tilde{\mathbf{r}}, \tilde{t})$. Here we recall that $\sigma=$ $\lambda / \lambda_{0}=\sqrt{\tau_{C} / \tau_{0}}$, leading to $\sigma \tau_{0} / \tau_{d}=\tau_{C} /\left(\sigma \tau_{d}\right)$, resulting in the cancellation of corresponding terms. In the limit $\sigma \ll 1$ one has

$$
\begin{aligned}
g_{\sigma \tilde{\mathbf{p}}}(\tilde{\mathbf{r}}, \tilde{t})= & -\frac{1}{1+\tau_{C} / \tau_{d}} \\
& \times\left[\tilde{\mathbf{p}} \cdot \partial_{\tilde{\mathbf{r}}} n_{0}(\tilde{\mathbf{r}}, \tilde{t}) \bar{f}_{\tilde{\mathbf{p}}}^{0}-\partial_{\tilde{\mathbf{r}}} \tilde{V}(\tilde{\mathbf{r}}, \tilde{t}) \cdot \partial_{\tilde{\mathbf{p}}} n_{0}(\tilde{\mathbf{r}}, \tilde{t}) \bar{f}_{\tilde{\mathbf{p}}}^{0}\right] .
\end{aligned}
$$

We note that $g_{\sigma \tilde{\mathbf{p}}}$ is an odd function of momentum and doesn't contribute to the density distribution. We further integrate over momentum the Eq. (B2) and note that the terms $\left\langle\tilde{\mathbf{p}} \cdot \partial_{\tilde{\mathbf{r}}} n_{0}(\tilde{\mathbf{r}}, \tilde{t}) \bar{f}_{\tilde{\mathbf{p}}}^{0}\right\rangle,\left\langle\partial_{\tilde{\mathbf{p}}} n_{0}(\tilde{\mathbf{r}}, \tilde{t}) \bar{f}_{\tilde{\mathbf{p}}}^{0}\right\rangle,\left\langle g_{\sigma \tilde{\mathbf{p}}}(\tilde{\mathbf{r}}, \tilde{t})\right\rangle$ are odd in $\mathbf{p}$ and thus vanish. The term $\left\langle\partial_{\tilde{\mathbf{p}}} g_{\sigma \tilde{\mathbf{p}}}(\tilde{\mathbf{r}}, \tilde{t})\right\rangle=0$ given that $g_{\sigma \tilde{\mathbf{p}}}(\tilde{\mathbf{r}}, \tilde{t})$ exponentially decays on the boundary of Brillouin zone. Hence, we get

$$
\partial_{\tilde{t}} n_{0}(\tilde{\mathbf{r}}, \tilde{t})\left\langle\bar{f}_{\tilde{\mathbf{p}}}^{0}\right\rangle+\left\langle\tilde{\mathbf{p}} \cdot \partial_{\tilde{\mathbf{r}}} g_{\sigma \tilde{\mathbf{p}}}(\tilde{\mathbf{r}}, \tilde{t})\right\rangle=0 .
$$

Plugging in $g_{\sigma \tilde{k}}(\tilde{\mathbf{r}}, \tilde{t})$ and performing the integration we reach at

$$
\partial_{\tilde{t}} n_{0}(\tilde{\mathbf{r}}, \tilde{t})=\frac{1}{1+\tau_{C} / \tau_{d}} \partial_{\tilde{\mathbf{r}}} \cdot\left[\partial_{\tilde{\mathbf{r}}} n_{0}(\tilde{\mathbf{r}}, \tilde{t})+\partial_{\tilde{\mathbf{r}}} \tilde{V}(\tilde{\mathbf{r}}, \tilde{t}) n_{0}(\tilde{\mathbf{r}}, \tilde{t})\right] .
$$

Now we recall that $n_{0}(\tilde{\mathbf{r}}, \tilde{t})=n(\tilde{\mathbf{r}}, \tilde{t}) e^{\frac{\tau_{0}}{\tau_{d}} \tilde{t}}$ and restore the original notations, resulting in Eq. (10).
[1] G. Wang, A. Chernikov, M. M. Glazov, T. F. Heinz, X. Marie, T. Amand, and B. Urbaszek, Rev. Mod. Phys. 90, 021001 (2018).

[2] M. Kulig, J. Zipfel, P. Nagler, S. Blanter, C. Schüller, T. Korn, N. Paradiso, M. M. Glazov, and A. Chernikov, Phys. Rev. Lett. 120, 207401 (2018).

[3] R. Perea-Causin, S. Brem, R. Rosati, R. Jago, M. Kulig, J. D. Ziegler, J. Zipfel, A. Chernikov, and E. Malic, Nano Lett. 19, 7317 (2019).

[4] M. M. Glazov, Phys. Rev. B 100, 045426 (2019).

[5] V. Shahnazaryan, O. Kyriienko, and H. Rostami, Phys. Rev. B 100, 165303 (2019).
[6] A. L. Ivanov, Europhys. Lett. 59, 586 (2002).

[7] A. G. Winbow, J. R. Leonard, M. Remeika, Y. Y. Kuznetsova, A. A. High, A. T. Hammack, L. V. Butov, J. Wilkes, A. A. Guenther, A. L. Ivanov, M. Hanson, and A. C. Gossard, Phys. Rev. Lett. 106, 196806 (2011).

[8] K. Cohen, R. Rapaport, and P. V. Santos, Phys. Rev. Lett. 106, 126402 (2011).

[9] F. Fedichkin, P. Andreakou, B. Jouault, M. Vladimirova, T. Guillet, C. Brimont, P. Valvin, T. Bretagnon, A. Dussaigne, N. Grandjean, and P. Lefebvre, Phys. Rev. B 91, 205424 (2015). 
[10] C. J. Dorow, Y. Y. Kuznetsova, J. R. Leonard, M. K. Chu, L. V. Butov, J. Wilkes, M. Hanson, and A. C. Gossard, Appl. Phys. Lett. 108, 073502 (2016).

[11] N. Peyghambarian, H. M. Gibbs, J. L. Jewell, A. Antonetti, A. Migus, D. Hulin, and A. Mysyrowicz, Phys. Rev. Lett. 53, 2433 (1984).

[12] C. Ciuti, V. Savona, C. Piermarocchi, A. Quattropani, and P. Schwendimann, Phys. Rev. B 58, 7926 (1998).

[13] V. Shahnazaryan, I. Iorsh, I. A. Shelykh, and O. Kyriienko, Phys. Rev. B 96, 115409 (2017).

[14] F. Barachati, A. Fieramosca, S. Hafezian, J. Gu, B. Chakraborty, D. Ballarini, L. Martinu, V. Menon, D. Sanvitto, and S. Kéna-Cohen, Nat. Nanotechnol. 13, 906 (2018).

[15] O. Bleu, G. Li, J. Levinsen, and M. M. Parish, Phys. Rev. Research 2, 043185 (2020).

[16] P. Stepanov, A. Vashisht, M. Klaas, N. Lundt, S. Tongay, M. Blei, S. Höfling, T. Volz, A. Minguzzi, J. Renard, C. Schneider, and M. Richard, Phys. Rev. Lett. 126, 167401 (2021).

[17] V. Shahnazaryan, V. K. Kozin, I. A. Shelykh, I. V. Iorsh, and O. Kyriienko, Phys. Rev. B 102, 115310 (2020).

[18] X. Zhu, P. B. Littlewood, M. S. Hybertsen, and T. M. Rice, Phys. Rev. Lett. 74, 1633 (1995).

[19] O. L. Berman, Y. E. Lozovik, D. W. Snoke, and R. D. Coalson, Phys. Rev. B 70, 235310 (2004).

[20] O. Kyriienko, E. B. Magnusson, and I. A. Shelykh, Phys. Rev. B 86, 115324 (2012).

[21] T. G. Pedersen, Phys. Rev. B 94, 125424 (2016).

[22] M. Engel and M. Steiner, in Proceedings of the LowDimensional Materials and Devices, edited by N. P. Kobayashi, A. A. Talin, and A. V. Davydov (SPIE, Bellingham, 2019), Vol. 11085, pp. 53-64.

[23] Y. Chen, Y. Huang, W. Lou, Y. Cai, and K. Chang, Phys. Rev. B 102, 165413 (2020).

[24] I. C. Gerber, E. Courtade, S. Shree, C. Robert, T. Taniguchi, K. Watanabe, A. Balocchi, P. Renucci, D. Lagarde, X. Marie, and B. Urbaszek, Phys. Rev. B 99, 035443 (2019).

[25] E. Lorchat, M. Selig, F. Katsch, K. Yumigeta, S. Tongay, A. Knorr, C. Schneider, S. Höfling, Phys. Rev. Lett. 126, 037401 (2021).

[26] D. Akinwande, C. J. Brennan, J. S. Bunch, P. Egberts, J. R. Felts, H. Gao, R. Huang, J.-S. Kim, T. Li, Y. Li, K. M. Liechti, N. Lu, H. S. Park, E. J. Reed, P. Wang, B. I. Yakobson, T. Zhang, Y.-W. Zhang, Y. Zhou, and Y. Zhu, Extreme Mech. Lett. 13, 42 (2017).

[27] J. H. Kim, J. H. Jeong, N. Kim, R. Joshi, and G.-H. Lee, J. Phys. D: Appl. Phys. 52, 083001 (2019).

[28] E. Khestanova, F. Guinea, L. Fumagalli, A. K. Geim, and I. V. Grigorieva, Nat. Commun. 7, 12587 (2016)

[29] E. Blundo, M. Felici, T. Yildirim, G. Pettinari, D. Tedeschi, A. Miriametro, B. Liu, W. Ma, Y. Lu, and A. Polimeni, Phys. Rev. Research 2, 012024(R) (2020).

[30] Y. Guo, B. Li, Y. Huang, S. Du, C. Sun, H. Luo, B. Liu, X. Zhou, J. Yang, J. Li, and C. Gu, Nano Res. 13, 2072 (2020).

[31] J. Feng, X. Qian, C.-W. Huang, and J. Li, Nat. Photon. 6, 866 (2012).

[32] A. Castellanos-Gomez, R. Roldan, E. Cappelluti, M. Buscema, F. Guinea, H. S. J. van der Zant, and G. A. Steele, Nano Lett. 13, 5361 (2013).
[33] T. P. Darlington, C. Carmesin, M. Florian, E. Yanev, O. Ajayi, J. Ardelean, D. A. Rhodes, A. Ghiotto, A. Krayev, K. Watanabe, T. Taniguchi, J. W. Kysar, A. N. Pasupathy, J. C. Hone, F. Jahnke, N. J. Borys, and P. J. Schuck, Nat. Nanotechnol. 15, 854 (2020).

[34] A. V. Tyurnina, D. A. Bandurin, E. Khestanova, V. G. Kravets, M. Koperski, F. Guinea, A. N. Grigorenko, A. K. Geim, and I. V. Grigorieva, ACS Photon. 6, 516 (2019).

[35] H. Luo, X. Li, Y. Zhao, R. Yang, L. Bao, Y. Hao, Y.-n. Gao, N. N. Shi, Y. Guo, G. Liu, L. Zhao, Q. Wang, Z. Zhang, G. Zhang, J. Sun, Y. Huang, H. Gao, and X. Zhou, Phys. Rev. Materials 4, 074006 (2020).

[36] W. Wu, L. Wang, Y. Li, F. Zhang, L. Lin, S. Niu, D. Chenet, X. Zhang, Y. Hao, T. F. Heinz, J. Hone, and Z. L. Wang, Nature 514, 470 (2014).

[37] H. Zhu, Y. Wang, J. Xiao, M. Liu, S. Xiong, Z. J. Wong, Z. Ye, Y. Ye, X. Yin, and X. Zhang, Nat. Nanotechnol. 10, 151 (2015).

[38] M. Droth, G. Burkard, and V. M. Pereira, Phys. Rev. B 94, 075404 (2016).

[39] H. Rostami, F. Guinea, M. Polini, and R. Roldán, npj 2D Mater. Appl. 2, 15 (2018).

[40] M. A. Cazalilla, H. Ochoa, and F. Guinea, Phys. Rev. Lett. 113, 077201 (2014).

[41] F. Guinea, M. I. Katsnelson, and A. K. Geim, Nat. Phys. 6, 30 (2010).

[42] O. Bistoni, P. Barone, E. Cappelluti, L. Benfatto, and F. Mauri, 2D Mater. 6, 045015 (2019).

[43] H. Rostami, A. G. Moghaddam, and R. Asgari, Phys. Rev. B 88, 085440 (2013).

[44] Y. Li, J. Ludwig, T. Low, A. Chernikov, X. Cui, G. Arefe, Y. D. Kim, A. M. van der Zande, A. Rigosi, H. M. Hill, S. H. Kim, J. Hone, Z. Li, D. Smirnov, and T. F. Heinz, Phys. Rev. Lett. 113, 266804 (2014).

[45] D. MacNeill, C. Heikes, K. F. Mak, Z. Anderson, A. Kormányos, V. Zólyomi, J. Park, and D. C. Ralph, Phys. Rev. Lett. 114, 037401 (2015).

[46] G. Aivazian, Z. Gong, A. M. Jones, R.-L. Chu, J. Yan, D. G. Mandrus, C. Zhang, D. Cobden, W. Yao, and X. Xu, Nat. Phys. 11, 148 (2015).

[47] A. Srivastava, M. Sidler, A. V. Allain, D. S. Lembke, A. Kis, and A. Imamoǧlu, Nat. Phys. 11, 141 (2015).

[48] H. Rostami and R. Asgari, Phys. Rev. B 91, 075433 (2015).

[49] H. Moon, G. Grosso, C. Chakraborty, C. Peng, T. Taniguchi, K. Watanabe, and D. Englund, Nano Lett. 20, 6791 (2020).

[50] A. V. Stier, N. P. Wilson, K. A. Velizhanin, J. Kono, X. Xu, and S. A. Crooker, Phys. Rev. Lett. 120, 057405 (2018).

[51] A. Jüngel, Transport Equations for Semiconductors, Lecture Notes in Physics Vol. 773 (Springer, Berlin, Heidelberg, 2009).

[52] E. Blundo, E. Cappelluti, M. Felici, G. Pettinari, and A. Polimeni, Appl. Phys. Rev. 8, 021318 (2021).

[53] Z. Dai, Y. Hou, D. A. Sanchez, G. Wang, C. J. Brennan, Z. Zhang, L. Liu, and N. Lu, Phys. Rev. Lett. 121, 266101 (2018).

[54] S. P. Timoshenko, Theory of Plates and Shells (McGraw Hill, India, 2010).

[55] L. D. Landau, L. P. Pitaevskii, A. M. Kosevich, and E. M. Lifshitz, Theory of Elasticity (Elsevier, Amsterdam, 1986).

[56] A. C. De Palma, G. Cossio, K. Jones, J. Quan, X. Li, and E. T. Yu, J. Vac. Sci. Technol. B 38, 042205 (2020). 
[57] F. Cadiz, C. Robert, E. Courtade, M. Manca, L. Martinelli, T. Taniguchi, K. Watanabe, T. Amand, A. C. H. Rowe, D. Paget, B. Urbaszek, and X. Marie, Appl. Phys. Lett. 112, 152106 (2018).

[58] L. Andreani, F. Tassone, and F. Bassani, Solid State Commun. 77, 641 (1991).

[59] M. Palummo, M. Bernardi, and J. C. Grossman, Nano Lett. 15, 2794 (2015).
[60] G. Moody, J. Schaibley, and X. Xu, J. Opt. Soc. Am. B 33, C39 (2016).

[61] L. Yuan, B. Zheng, J. Kunstmann, T. Brumme, A. B. Kuc, C. Ma, S. Deng, D. Blach, A. Pan, and L. Huang, Nat. Mater. 19, 617 (2020).

[62] E. Blundo, C. Di Giorgio, G. Pettinari, T. Yildirim, M. Felici, Y. Lu, F. Bobba, and A. Polimeni, Adv. Mater. Interfaces 7, 2000621 (2020). 